\title{
Status of multi-beam long trace-profiler development
}

Mikhail V. Gubarev, Kiranmayee Kilaru, Brian Ramsey, NASA Marshall Space Flight Ctr. Wayne R. McKinney, Daniel J. Merthe, Valeriy V. Yashchuk, Lawrence Berkeley National Lab. Peter Z. Takacs, Brookhaven National Lab.

\author{
$\underline{\text { Abstract }}$ \\ The multi-beam long trace profiler (MB-LTP) is under development at NASA's Marshall Space \\ Flight Center. The traditional LTPs scans the surface under the test by a single laser beam \\ directly measuring the surface figure slope errors. While capable of exceptional surface slope \\ accuracy, the LTP single beam scanning has slow measuring speed. Metrology efficiency can be \\ increased by replacing the single laser beam with multiple beams that can scan a section of the \\ test surface at a single instance. The increase in speed with such a system would be almost \\ proportional to the number of laser beams. The progress for a multi-beam long trace profiler \\ development will be presented.
}

